\title{
Organized vs. Competitive Corruption
}

\author{
MARCO CELENTANI* \\ Universidad Carlos III de Madrid, Spain \\ JUAN-JOSÉ GANUZA \\ Universitat Pompeu Fabra, Spain
}

celentan@eco.uc3m.es

juanjo.ganuza@econ.upf.es

\begin{abstract}
We study bureaucratic corruption in a model in which a constituency sets required levels for a given set of activities. Each activity is carried out by an external provider and its realization is supervised by a bureaucrat. Bureaucrats are supposed to act on behalf of the constituency, but they can allow providers to deliver lower activity levels than contracted in exchange for a bribe. Given this, the constituency sets the optimal activity levels weighing off the value of activity levels, their costs, as well as the possibility for the bureaucrats to be corrupt. We use this setup to study the impact on equilibrium corruption of the degree of decentralization of corruption. To do this we compute equilibrium corruption in two different settings: (1) Each bureaucrat acts in such a way as to maximize his own individual utility (competitive corruption); (2) An illegal syndicate oversees the corruption decisions of the population of bureaucrats in such a way as to maximize total proceeds from corruption (organized corruption). We show that the illegal syndicate acts in such a way as to restrain the total number of corrupt transactions and corruption is lower when it is organized than when it is competitive.
\end{abstract}

Keywords: competitive and organized corruption, institutional response

AMS subject classifi ation: 91A10, 91A80, 91B26

\section{Introduction}

Corruption has long been recognized as one of the most important factors affecting the creation and the distribution of wealth and the last years have witnessed an increased interest in the topic. Innumerable accounts of corruption cases have received wide exposure in the press, and the concern of the international economic community is revealed by the efforts that international organizations and consulting organizations devote to the problem. The OECD's “Convention on Combating Bribery of Foreign Public Off cials in International Business Transactions" and other projects of the World Trade Organization, the United Nations, the Council of Europe are but a few examples of supranational attempts to f ght corruption. Several consulting organizations, including the Business International Corporation, part of the Economist Intelligence Unit, and International Country Risk Guide, part of the PRS Group, provide rankings of countries' institutional eff ciency, including bureaucratic eff ciency and corruption.

* Corresponding author: Department of Economics, Universidad Carlos III de Madrid, Getafe (Madrid) 28903, Spain. 
The economic profession has increased its attention to the problem especially since it has become clear that corruption does not simply lead to redistribution of surplus, but, due to its illegal nature, also creates a score of additional distortions in the production of wealth. Early contributions to this line of research include Tullock's [16] and Krueger's [9] studies of the distortions implied by rent-seeking activities. Mauro [12,13] uses corruption indices to show corruption's impact on investment levels, government expenditure composition, and ultimately on GDP growth.

Recent research has analyzed the different ways corrupt transactions are arranged ranging from the substantially centralized networks of Italy, the Soviet Union, South Korea and Japan to the more decentralized systems operating in former Soviet republics, India and Africa.

An organized corruption network is likely to reduce transaction costs of possibly complex corrupt agreements, as documented by Cartier-Bresson [3] and discussed by Goudie and Stasavage [8]. But the economic profession has also indicated that it can serve as a means to limit the erosion of the corruption revenue base.

In this line of research Shleifer and Vishny [15] argued that when different government assets awarded by corrupt bureaucrats are complements, the bribe sought by any one bureaucrat increases the cost of the asset and imposes pecuniary externalities on the other corrupt bureaucrats by decreasing the demand for their assets and ultimately their ability to prof $t$ from corruption. Shleifer and Vishny [15] argue that if bureaucrats collude in bribe setting, they internalize the external effects to maximize total corruption revenue. In the same line Bardhan [2] argued that centralization of bribe setting also allows "lump sum" corruption that minimizes distortions and the eff ciency cost of corruption.

The goal of this paper is to focus on an additional channel through which an organized network can reduce the erosion of the corruption revenue base. We argue that, if society optimally responds to the existing level of corruption, a higher degree of centralization of corruption decisions leads to lower corruption levels. We focus on two alternative setups, one in which each bureaucrat decides individually whether to be corrupt or not, a situation we refer to as competitive corruption, and the other in which the decision of how many corrupt transactions are carried out is taken by an illegal syndicate maximizing the total net proceeds of corrupt transactions, a situation we refer to as organized corruption.

We propose a game-theoretical model in which a constituency has to set required levels for a given set of activities (examples include procurement, regulation, tax collection). Each activity is carried out by an external party, called a provider, and its realization is supervised by a bureaucrat. Each bureaucrat is supposed to act on behalf of the constituency, but he can allow the provider to deliver a lower activity level than contracted in exchange for a bribe. Given this, the constituency sets the optimal activity levels weighing off the value of activities, their costs, as well as the possibility for the bureaucrats to be corrupt.

We study a situation in which a bureaucrat who intends to be corrupt has to bear a "corruption entry cost" before the constituency sets the required activity levels and 
in anticipation of them. We believe that this sequential specif cation is especially well suited to study corruption in that it recognizes there is a moment in which some corruption costs have already been sunk and cannot be recovered even if the involved parties decide to pull off a corrupt transaction. Two reasons why this might be the case are the following:

1. Corrupt transactions are often suff ciently complicated to require early costly arrangements from involved parties.

2. An individual bureaucrat's cost of arranging corrupt transactions decreases with experience and a rational bureaucrat should interpret part of the costs borne in the early transactions as irrecoverable investments that make later corrupt transactions possible.

Because average corruption payoff is increasing in the activity levels set by the constituency, and given the constituency's response to higher levels of corruption is to reduce required activity levels, in the subgame perfect Nash equilibrium of the organized corruption game the illegal syndicate acts in such a way as to restrain the total number of corrupt transactions, so that corruption is lower when it is organized than when it is competitive.

As opposed to other research in the feld, our paper makes an explicit attempt to characterize the constituency's optimal behavior and how it depends on the degree of centralization of corruption. The syndicate's restrained equilibrium behavior mentioned above has important policy implications for the constituency, as it implies that it is optimal for it to choose higher activity levels when corruption is organized than when it is competitive. In the case of procurement of a good with a quality parameter to be set by the procurement agency, our results imply that the procurement principal should choose a higher quality level if corruption is organized.

Our result is similar to Shleifer and Vishny's [15] in that we also suggest that organized corruption can serve to curb the negative externalities individual bureaucrats impose on each other. While Shleifer and Vishny [15] claim that these externalities arise because the willingness to pay for corrupt acts is decreasing in corruption, we propose the institutional response to the existing levels of corruption as an additional avenue through which these externalities might arise.

Apart from the previous similarity a major difference between the testable implications of our model and the ones of Shleifer and Vishny [15] exists. Our model predicts that, as compared to competitive corruption, under organized corruption total corruption is lower and the unit bribe is higher, while Shleifer and Vishny's [15] predictions are exactly the opposite. Although we regard the two models as possibly complementary, the differences in their testable implications can be used to assess the relative merits of their assumptions.

As mentioned above this paper focuses on the institutional optimal response to corruption, following a renewed interest in the issue. Laffont and N'Guessan [10] con- 
sider a model of regulation in which the agent may be corrupt and in which the regulator optimally chooses the contract to be offered to the regulated $\mathrm{frm}$ and to the agent. Ades and di Tella [1] propose a model in which a regulation agent may be corrupt and argue that an increase in competition parameters that lowers regulated f rms' equilibrium prof ts has an ambiguous effect on the level of corruption due to the regulator's optimal response that changes the agent's wage and therefore his incentives to be corrupt.

In a related paper, Celentani and Ganuza [5], we propose a procurement problem in which the procurement agent is supposed to allocate the realization of a project according to a competitive mechanism that values bids in terms of the proposed price and quality. In a similar vein as in this paper, the procurement agent can decide to be corrupt and allow an arbitrary f rm to be awarded the realization of the project and to produce a quality level lower than the announced and the principal can select an appropriate competitive mechanism given the anticipated corruption level. We study the impact on corruption of the increased competitiveness of the environment and show that, because of the regulatory response of the principal and contrary to conventional wisdom, corruption may well be increasing in competition.

The paper is structured in the following way. Section 2 presents the model. Section 3 derives the equilibrium in the competitive setting. In section 4 we discuss organized corruption and we compare the equilibrium levels of corruption and activity levels with the case of competitive corruption. Section 5 concludes.

\section{The model}

Consider a situation in which a constituency employs a population of bureaucrats with mass 1 . Each bureaucrat is in charge of overseeing a particular activity that is to be carried out by an external party, to whom we refer as a provider. The constituency values a project carried out at level $\lambda$ and with price $p$ according to the following utility function, $\mathcal{U}^{\mathcal{C}}(\lambda, p)=V(\lambda)-p$. We assume the provider is risk-neutral, that it is common knowledge that the provider has a cost of realizing activity level $\lambda$ equal to $C(\lambda)$ and we normalize its reservation level to zero.

This kind of situation is meant to represent a wide range of possible instances in which corruption may take place, with examples stretching from procurement ${ }^{1}$ (where $\lambda$ is the quality of the good or service to be procured) to regulation (with $\lambda$ representing a reduction of carbon dioxide emissions) to tax collection (with $\lambda$ standing for a company's employment of minority workers that entitles it to a tax rebate).

${ }^{1}$ The purpose of looking at such a simplif ed setting is to avoid unnecessary complications. The reader interested in a more realistic description of this kind of problem is referred to Celentani and Ganuza [5] in which we analyze a procurement problem with a f nite number of potential contractors and asymmetric information on their cost functions. The model we present here retains all the relevant features of this more realistic setting while simplifying the derivation of the results. 
In the following we will consider payoff and cost functions that satisfy the following conditions:

\section{Assumption 1.}

(1) $V(\cdot)$ is twice continuously differentiable,

$$
V^{\prime}(\cdot)>0, \quad V^{\prime \prime}(\cdot)<0, \quad \lim _{\lambda \rightarrow 0^{+}} V^{\prime}(\lambda)=+\infty, \quad \lim _{\lambda \rightarrow+\infty} V^{\prime}(\lambda)=0 .
$$

(2) $C(\cdot)$ is twice continuously differentiable,

$$
C^{\prime}(\cdot)>0, \quad C^{\prime \prime}(\cdot) \geqslant 0, \quad \lim _{\lambda \rightarrow 0^{+}} C^{\prime}(\lambda)<+\infty .
$$

Bureaucrats are risk-neutral and each bureaucrat can verify whether the delivered activity level he oversees coincides with the contracted level, but he can also illegally allow the provider to deliver a lower activity level, $\lambda_{c}$, in exchange for a bribe, a choice we will refer to as arranging a corrupt transaction.

In order to be able to arrange a corrupt transaction a bureaucrat has to pay an idiosyncratic corruption entry cost, $\beta$, prior to the constituency announcing $\lambda$. To simplify the presentation we will assume that $\beta$ is uniformly distributed on $[0,1]$. If a bureaucrat pays the corruption entry cost and then arranges a corrupt transaction, he is detected with probability $\mu \in(0,1)$ and in this case he and the provider are imposed penalties, $P^{\mathcal{B}}>0$ and $P^{\mathcal{P}}>0$, respectively. The detection probability $\mu$ and the penalties $P^{\mathcal{B}}$ and $P^{\mathcal{P}}$ are thought of as features of the legal framework and in the following will therefore be assumed to be exogenously given. If a bureaucrat does not arrange a corrupt transaction, or if he does but he is not detected, he is paid a f xed wage which is normalized to zero.

As was already brief y mentioned in the introduction, the assumption that a bureaucrat who intends to arrange a corrupt transaction has to pay a corruption entry cost before the constituency is allowed to set activity levels is justif able for two independent and complementary reasons.

1. Corrupt transactions often need important arrangements that need to be made with suff cient anticipation (e.g., establishing an appropriate network). In other words, if it is reasonable to think that certain details on the transaction will be sorted out in the end, e.g., the payment the bureaucrat is entitled to, there are other arrangements that cannot be delayed until the last moment and that require costly provisions.

2. Assuming that bureaucrats have to pay a corruption entry cost at the beginning of the game implies that there is a moment in which bureaucrats' decisions to arrange a corrupt transaction or not are not very responsive to the constituency's choices. This effect is meant to describe the high degree of irreversibility of individual decisions to be corrupt. In other words, being corrupt at any given date lowers the (opportunity) cost of being corrupt in the future of a long-lived bureaucrat, ${ }^{2}$ and at any given date

\footnotetext{
2 This assumption is often used in the corruption literature and has been documented for criminal behavior.
} 
the bureaucrats that have been corrupt in the past have already sunk a corruption entry $\operatorname{cost}^{3}$ and are therefore not very responsive to the constituency's choices affecting the prof tability of corruption.

The results of the simplif ed and somewhat extreme setup described above can be shown to hold so long a positive fraction of agents has to decide whether to pay the corruption entry cost prior to the constituency setting the required activity levels.

Given all of the above, we choose to analyze competitive corruption by making use of the extensive form summarized in the following:

1. (Stage 1) Each bureaucrat decides whether to pay the corruption entry cost $\beta$ that will then enable him to be corrupt.

2. (Stage 2) The constituency publicly announces the required activity level $\lambda$.

3. (Stage 3) If a bureaucrat has not paid the corruption entry cost $\beta$ in stage 1 , he can only instruct the provider to deliver the required activity level and reimburse it the cost of producing it, $C(\lambda){ }^{4}$

If a bureaucrat has paid the corruption entry $\operatorname{cost} \beta$ in stage 1 , he can decide to arrange a corrupt transaction and in this case he bargains with the provider over a bribe $b$ to be received in exchange for the agreement that the provider will be permitted to deliver a lower activity level, $\lambda_{c}<\lambda$, and receive a cost reimbursement $C(\lambda)$.

4. With probability $\mu$ the constituency gets a perfect signal of delivered activity level. If the delivered level is less than the required one, the bureaucrat and the provider are imposed penalties $P^{\mathcal{B}}$ and $P^{\mathcal{P}}$.

The equilibrium concept we use is subgame perfect Nash equilibrium.

\section{Competitive corruption}

This section is devoted to the characterization of equilibrium for the extensive form given above describing corruption in a competitive setting.

Given we are interested in subgame perfect Nash equilibria we will proceed as follows. In section 3.1 we will focus on stage 3 and characterize the best response of the bureaucrats to a given activity level $\lambda$ set by the constituency in stage 2 . Section 3.2 studies stage 2 and focuses on the best response of the constituency to a given $\gamma$ (fraction of agents who paid the corruption entry cost in stage 1) given the stage 3 best response of bureaucrats to a given $\lambda$ derived in the previous subsection. In section 3.3 we will turn to stage 1 and study how individual bureaucrats make the decision to pay the corruption entry cost or not. Section 3.4 combines the previous results to characterize the equilibrium.

${ }^{3}$ A bureaucrat who decides to be corrupt typically sinks a f xed cost to arrange a network of contacts that can then be used for several transactions.

${ }^{4}$ Recall that the $\mathrm{frm}$ 's reservation level is assumed to be 0 . 


\subsection{Stage 3: Arranging a corrupt transaction and bargaining over a bribe}

If a bureaucrat has paid the corruption entry $\operatorname{cost} \beta$ in stage 1 , after observing the activity level $\lambda$ set by the constituency in stage 2 , in stage 3 he is in a position to arrange a corrupt transaction and to bargain with the provider over the bribe to be received in exchange for the fact that the provider will be paid $C(\lambda)$ but will be asked to deliver only $\lambda_{c}<\lambda .^{5}$

We assume that the outcome of the bargaining process between the corrupt bureaucrat and the provider is the solution to a generalized Nash bargaining problem. Let $\pi^{\mathcal{B}}(\lambda)$ denote the agreement payoff to a corrupt bureaucrat when the activity level set by the constituency is $\lambda$ and $d^{\mathcal{B}}$ his disagreement point. Let $\pi^{\mathcal{P}}(\lambda)$ denote the agreement payoff from a corrupt transaction to the provider when the activity level set by the constituency is $\lambda$ and let $d^{\mathcal{P}}$ denote the provider's disagreement point. For a given activity level $\lambda$, the equilibrium bribe $b(\lambda)$ will then be given by the solution to the following problem

$$
\max _{b(\lambda):\left(\pi^{\mathcal{B}}, \pi^{\mathcal{P}}\right) \geqslant\left(d^{\mathcal{B}}, d^{\mathcal{P}}\right)}\left(\pi^{\mathcal{B}}-d^{\mathcal{B}}\right)^{\alpha}\left(\pi^{\mathcal{P}}-d^{\mathcal{P}}\right)^{1-\alpha} .
$$

A type $\beta$ corrupt bureaucrat's agreement payoff is

$$
\pi^{\mathcal{B}}=b(\lambda)-\mu P^{\mathcal{B}},
$$

i.e., bribe minus expected penalty $;{ }^{6}$ his disagreement level will simply be his constant reservation wage which has been normalized to zero, $d_{B}=0$. Given activity level $\lambda$, the provider's agreement payoff is

$$
\pi^{\mathcal{P}}=C(\lambda)-C\left(\lambda_{c}\right)-b(\lambda)-\mu P^{\mathcal{P}},
$$

i.e., the payment it receives, minus the cost of producing activity level $\lambda_{c}$, minus the bribe, minus the expected penalty; its disagreement point will be its payoff without corruption, $d^{\mathcal{P}}=0$. Given this, it is easy to show that the equilibrium bribe paid by the provider to the bureaucrat is

$$
b^{*}(\lambda)=\alpha\left[C(\lambda)-C\left(\lambda_{c}\right)-\mu P^{\mathcal{P}}\right]+(1-\alpha) \mu P^{\mathcal{B}} .
$$

Substituting (2) into (1) we get the Nash bargaining equilibrium payoffs for the bureaucrat and the provider

$$
\begin{aligned}
& \pi^{\mathcal{B} *}(\lambda)=\alpha\left[C(\lambda)-C\left(\lambda_{c}\right)-\mu\left(P^{\mathcal{P}}+P^{\mathcal{B}}\right)\right]=\alpha[C(\lambda)-k], \\
& \pi^{\mathcal{P} *}(\lambda)=(1-\alpha)\left[C(\lambda)-C\left(\lambda_{c}\right)-\mu\left(P^{\mathcal{P}}+P^{\mathcal{B}}\right)\right]=(1-\alpha)[C(\lambda)-k],
\end{aligned}
$$

where we let $k=C\left(\lambda_{c}\right)+\mu\left(P^{\mathcal{B}}+P^{\mathcal{P}}\right)>0$. It is immediate to see that if

$$
C(\lambda)-k>0,
$$

${ }^{5}$ In terms of the procurement example we referred to above, $\lambda$ can be interpreted as the quality of a given product or service to be supplied and $C(\lambda)$ the cost of supplying it, with $\lambda_{c}$ and $C\left(\lambda_{c}\right)$ representing, respectively, the quality supplied under corruption and the cost of supplying it.

${ }^{6}$ Notice that the corruption entry cost $\beta$ is paid before entering the bargaining stage. Being a sunk cost, it is paid regardless of whether an agreement is reached or not and it is to be disregarded in computing agreement and disagreement payoffs. 
i.e., if corruption creates a strictly positive surplus to be shared, $\pi^{\mathcal{B} *}>d^{\mathcal{B}}=0$ and $\pi^{\mathcal{P} *}>d^{\mathcal{P}}=0$ both hold and $b(\lambda)$ is increasing in the bureaucrat's bargaining power $\alpha$. When

$$
C(\lambda)-k \leqslant 0,
$$

i.e., when the surplus to be shared in the bargaining is nonpositive, no corrupt transaction will be arranged and therefore no bargaining will take place.

Letting $\lambda_{\min }$ be such that $C\left(\lambda_{\min }\right)-k=0$, one can summarize the previous observations by saying that if $\lambda>\lambda_{\min }$, each bureaucrat who had paid the corruption entry cost in stage 1 arranges a corrupt transaction in stage 3 , as he will $\mathrm{f}$ nd it strictly profitable, and when $\lambda \leqslant \lambda_{\min }$, no bureaucrat will arrange a corrupt transaction in stage 3 , as it is strictly unprof table.

\subsection{Stage 2: The optimal activity level}

Consider a situation in which the constituency faces a continuum of bureaucrats with unit mass and suppose the constituency knows that a fraction $\gamma$ of them has paid the corruption entry cost. Given the constituency does not know the identity of the bureaucrats that have paid the corruption entry cost (i.e., that are in a position to be corrupt) this is equivalent to believing that any particular bureaucrat has paid the corruption entry cost with probability $\gamma$. Suppose the constituency sets an activity level $\lambda$. If a bureaucrat had not paid the corruption entry cost in stage 1 , he can only be honest and guarantee that the delivered activity level is the required while paying the provider a price equal to the cost of delivering $\lambda, C(\lambda)$. If a bureaucrat had paid the corruption entry cost in stage 1 , from the previous section we know that he will arrange a corrupt transaction with the provider if and only if $\lambda>\lambda_{\min }$; in this case he would require only $\lambda_{c}$ of the provider, he would announce that the delivered activity level is $\lambda$ and would accordingly pay $C(\lambda)$ to the provider. If on the other hand $\lambda \leqslant \lambda_{\min }$, a corrupt transaction generates a nonpositive surplus for the bureaucrat and the provider and it would therefore not take place.

Given this and because all activities are ex ante identical for the constituency, the activity level set by the constituency for each bureaucrat is the solution to the following maximization problem:

$$
f(\gamma)=\max \left\{\max _{\lambda \leqslant \lambda_{\min }} V(\lambda)-C(\lambda), \max _{\lambda>\lambda_{\min }}(1-\gamma) V(\lambda)+\gamma V\left(\lambda_{c}\right)-C(\lambda)\right\} .
$$

The second term in the external maximand is the maximized utility of the constituency when $\lambda>\lambda_{\min }$, i.e., in the set of values of $\lambda$ that imply that all the bureaucrats that paid the corruption entry cost (mass $\gamma$ ) arrange a corrupt transaction as they f nd it prof table (therefore a fraction $\gamma$ of the activities are carried out at the level $\lambda_{c}$ ); the frst term is instead the maximized utility of the constituency when $\lambda \leqslant \lambda_{\min }$, i.e., in the set of values of $\lambda$ that imply that all the bureaucrats that paid the corruption entry cost give up the option of arranging a corrupt transaction as they f nd it unprof table (no corrupt transaction would be arranged). 
Let $\bar{\lambda}(\gamma)$ be implicitly def ned by the f rst-order condition of the second maximization problem in the external maximand in (6) above ${ }^{7}$

$$
(1-\gamma) V^{\prime}(\bar{\lambda}(\gamma))=C^{\prime}(\bar{\lambda}(\gamma))
$$

and let $\lambda_{\mathrm{NC}}=\bar{\lambda}(0)>0$, i.e., def ne $\lambda_{\mathrm{NC}}$ as the optimal activity level for the constituency when no bureaucrat paid the corruption cost in stage 1. Notice that under assumption 1, $\bar{\lambda}(\gamma)$ exists, is unique, positive, and f nite and that

$$
\bar{\lambda}^{\prime}(\gamma)=\frac{V^{\prime}(\bar{\lambda}(\gamma))}{(1-\gamma) V^{\prime \prime}(\bar{\lambda}(\gamma))-C^{\prime \prime}(\bar{\lambda}(\gamma))}<0 .
$$

Notice also that (8) together with $\bar{\lambda}(1)=0$ and the continuity of $\bar{\lambda}(\gamma)$ imply that when $\lambda_{\mathrm{NC}}>\lambda_{\min }$ there exists a $\gamma^{\prime} \in(0,1)$ such that $\bar{\lambda}\left(\gamma^{\prime}\right)=\lambda_{\min }$ and $\bar{\lambda}(\gamma)>\lambda_{\min }$ if and only if $\gamma<\gamma^{\prime}$.

The following function refers to a situation in which $\bar{\lambda}(\gamma)>\lambda_{\min }$, i.e., $\gamma \in\left[0, \gamma^{\prime}\right)$, and describes the excess payoff for the constituency from setting $\lambda=\bar{\lambda}(\gamma)$ (and accept that the mass of bureaucrats who have already paid the corruption entry cost will carry out corrupt transactions) rather than $\lambda=\lambda_{\min }$ (and in this way have no corrupt transactions arranged in stage 3 )

$$
h(\gamma)=(1-\gamma) V(\bar{\lambda}(\gamma))+\gamma V\left(\lambda_{c}\right)-C(\bar{\lambda}(\gamma))-\left(V\left(\lambda_{\min }\right)-C\left(\lambda_{\min }\right)\right) .
$$

Given $h(\gamma)$ is continuous for all $\gamma \in\left[0, \gamma^{\prime}\right), h(0)>0, h\left(\gamma^{\prime}\right)<0$, and, from the envelope theorem, $h^{\prime}(\gamma)=V(\bar{\lambda}(\gamma))-V\left(\lambda_{c}\right)<0, h(\gamma)$ has a unique zero, $\tilde{\gamma} \in\left[0, \gamma^{\prime}\right)$.

For the following it is also useful to def ne $\Sigma(S)$ as the set of probability distributions over $S \subseteq R_{+}$.

Given all of the above we are in a position to summarize the constituency's best response to a given fraction $\gamma$ of bureaucrats having paid the corruption entry cost, $\lambda(\gamma)$, as follows:

Lemma 1. The best response correspondence of the constituency to a given fraction $\gamma$ of bureaucrats having paid the corruption entry cost, $\lambda(\gamma)$, is

(1) If $\lambda_{\mathrm{NC}} \leqslant \lambda_{\min }$,

$$
\lambda(\gamma)=\lambda_{\mathrm{NC}} .
$$

(2) If $\lambda_{\mathrm{NC}}>\lambda_{\min }$,

$$
\lambda(\gamma)= \begin{cases}\bar{\lambda}(\gamma) & \text { if } \gamma \in[0, \tilde{\gamma}), \\ \Sigma\left(\left\{\lambda_{\min }, \bar{\lambda}(\gamma)\right\}\right) & \text { if } \gamma=\widetilde{\gamma}, \\ \lambda_{\min } & \text { if } \gamma \in(\widetilde{\gamma}, 1] .\end{cases}
$$

${ }^{7}$ The second order condition $(1-\gamma) V^{\prime \prime}(\bar{\lambda}(\gamma))-C^{\prime \prime}(\bar{\lambda}(\gamma))<0$ is satisfi d since $(1-\gamma)>0, V^{\prime \prime}(\bar{\lambda}(\gamma))<0$ and $C^{\prime \prime}(\bar{\lambda}(\gamma))>0$. 
Proof. Given (8) implies that $\bar{\lambda}(\gamma)<\bar{\lambda}(0)=\lambda_{\mathrm{NC}}$ for all $\gamma \in(0,1]$, assumption 1 implies that setting $\lambda>\lambda_{\mathrm{NC}}$, is inferior to setting $\lambda=\lambda_{\mathrm{NC}}$ for $\gamma \in[0,1]$. This, together with assumption 1 implies that we can restrict attention to $\lambda \in\left(0, \lambda_{\mathrm{NC}}\right]$.

(1) When $\lambda_{\mathrm{NC}} \leqslant \lambda_{\min }$ no corrupt transaction is arranged in stage 3 for $\lambda \in\left(0, \lambda_{\mathrm{NC}}\right]$. Given we can restrict attention to $\lambda \in\left(0, \lambda_{\mathrm{NC}}\right]$, and given $\bar{\lambda}(0)=\lambda_{\mathrm{NC}}$ is the optimum level of $\lambda$ when no corrupt transaction is expected in stage 3 , the optimum for the constituency is $\lambda=\lambda_{\mathrm{NC}}$.

(2) Recall we can restrict attention to $\lambda \in\left(0, \lambda_{\mathrm{NC}}\right]$. When $\lambda_{\mathrm{NC}}>\lambda_{\min }$ we can partition the set $\left(0, \lambda_{\mathrm{NC}}\right]$ into $\left(0, \lambda_{\min }\right]$ and $\left(\lambda_{\min }, \lambda_{\mathrm{NC}}\right]$.

Consider f rst $\lambda \in\left(0, \lambda_{\min }\right]$. For all $\lambda \in\left(0, \lambda_{\min }\right]$ none of the bureaucrats who paid the corruption entry cost in stage 1 will arrange a corrupt transaction in stage 3 ; Given $\lambda_{\mathrm{NC}}>\lambda_{\min }$, and $V(\lambda)-C(\lambda)$ is increasing in $\lambda$ for $\lambda \in\left[0, \lambda_{\mathrm{NC}}\right)$, for any $\gamma$, the optimal value of $\lambda$ in $\left(0, \lambda_{\min }\right]$ is $\lambda_{\min }$.

Consider now $\lambda \in\left(\lambda_{\min }, \lambda_{\mathrm{NC}}\right]$. For all $\lambda \in\left(\lambda_{\min }, \lambda_{\mathrm{NC}}\right)$ all bureaucrats who paid the corruption entry cost in stage 1 will be corrupt in stage 3 , and this implies, that for a given $\gamma$, the optimal value of $\lambda$ in this set is $\bar{\lambda}(\gamma)$.

The previous two observations imply that the best response of the constituency to a given $\gamma$ can only be $\lambda_{\min }, \bar{\lambda}(\gamma)$ or a probability distribution over them. The rest of the proof considers a partition of the set of possible value of $\gamma,[0,1]$, and provides the best response in each element of the partition, thus concluding the proof of (2). For the following recall that $0<\tilde{\gamma}<\gamma^{\prime}<1$.

(1) For $\gamma \in[0, \tilde{\gamma}), h(\gamma)>0$ implies that $\bar{\lambda}(\gamma)$ gives the constituency a higher payoff than $\lambda_{\min }$ and the best response of the constituency is therefore $\bar{\lambda}(\gamma)$.

(2) For $\gamma=\tilde{\gamma}, h(\gamma)=0$ implies that $\bar{\lambda}(\gamma)$ and $\lambda_{\text {min }}$ give the constituency the same payoff and the best response of the constituency is therefore any probability distribution with support $\left\{\lambda_{\min }, \bar{\lambda}(\gamma)\right\}$.

(3) For $\gamma \in(\tilde{\gamma}, 1], h(\gamma)<0$ implies that $\lambda_{\text {min }}$ gives the constituency a higher payoff than $\bar{\lambda}(\gamma)$ and the best response of the constituency is therefore $\lambda_{\min }$.

\subsection{Stage 1: Paying corruption entry costs}

From section 3.1 if a bureaucrat in stage 1 believes that the constituency will set activity level $\lambda>\lambda_{\min }$ in stage 2 , he anticipates a gross equilibrium payoff of $\pi^{\mathcal{B} *}(\lambda)$ from arranging a corrupt transaction in stage 3 and will therefore pay the corruption entry $\operatorname{cost} \beta$ if and only it is at least compensated by the gross payoff $\pi^{\mathcal{B} *}(\lambda)$, i.e., if and only if

$$
\beta \leqslant \pi^{\mathcal{B} *}(\lambda)=\alpha[C(\lambda)-k] .
$$

If a bureaucrat in stage 1 believes that the constituency will set activity level $\lambda \leqslant \lambda_{\min }$ in stage 2 , he anticipates a gross equilibrium payoff of 0 in stage 3 and will therefore choose not to pay the corruption entry $\operatorname{cost} \beta$. The previous arguments can be summarized by saying the if the bureaucrats believe that the activity level that will be 
set by the constituency in stage 2 is $\lambda$, the set of types who choose to pay the corruption entry cost at stage 1

$$
\widetilde{\beta}(\lambda)= \begin{cases}{[0,1]} & \text { if } \lambda \geqslant \lambda_{\min } \text { and } \alpha(C(\lambda)-k) \geqslant 1, \\ {[0, \alpha(C(\lambda)-k)]} & \text { if } \lambda \geqslant \lambda_{\min } \text { and } \alpha(C(\lambda)-k)<1, \\ \emptyset & \text { if } \lambda<\lambda_{\text {min }} .\end{cases}
$$

Notice that in the decentralized case we are describing in this section each bureaucrat has 0 mass and this implies that no bureaucrat has incentives to play in a strategic way exploiting the frst-mover advantage and that his decision to be corrupt or not only depends on whether being corrupt is prof table for him or not. ${ }^{8}$ Given this and because $\beta$ has been assumed to be uniformly distributed on $[0,1]$, the mass of bureaucrats who pay the corruption entry cost when they anticipate a given activity level $\lambda, \gamma(\lambda)$, is

$$
\gamma(\lambda)= \begin{cases}1 & \text { if } \lambda \geqslant \lambda_{\min } \text { and } \alpha(C(\lambda)-k) \geqslant 1, \\ \alpha(C(\lambda)-k) & \text { if } \lambda \geqslant \lambda_{\min } \text { and } \alpha(C(\lambda)-k)<1, \\ 0 & \text { if } \lambda<\lambda_{\min } .\end{cases}
$$

For $\lambda>\lambda_{\min }, \gamma^{\prime}(\cdot)>0$ and the mass of corrupt bureaucrats is increasing in the anticipated activity level set by the constituency.

\subsection{Equilibrium}

The equilibrium under competitive corruption is obtained combining equations (7) and (11) representing the best response of the constituency to the stage 1 aggregate strategy of the bureaucrats and the stage 1 aggregate best response of the bureaucrats to the anticipated activity level set by the constituency in stage 2 , respectively.

The equilibrium is def ned by the following two equations:

$$
\begin{aligned}
& \lambda=\lambda(\gamma) \\
& \gamma=\gamma(\lambda) .
\end{aligned}
$$

Proposition 2. A unique equilibrium $(\widehat{\gamma}, \widehat{\lambda})$ exists.

(1) If $\lambda_{\mathrm{NC}}>\lambda_{\min }, \widehat{\gamma} \in(0,1)$ and $\widehat{\lambda} \in\left(0, \lambda_{\mathrm{NC}}\right)$.

(2) If $\lambda_{\mathrm{NC}} \leqslant \lambda_{\min }, \widehat{\gamma}=0$ and $\widehat{\lambda}=\lambda_{\mathrm{NC}}$.

Proof. (1) First suppose that an equilibrium exists in which $\widehat{\gamma}>\tilde{\gamma}$. This implies that $\widehat{\lambda}=\lambda_{\text {min }}$ which in turn implies that $\widehat{\gamma}=0$, a contradiction. This implies that there can only exist equilibria with $\widehat{\gamma} \leqslant \tilde{\gamma}$.

Let now

$$
g(\gamma)=\gamma-\alpha(C(\bar{\lambda}(\gamma))-k)
$$

${ }^{8}$ In section 4 we will turn to the case in which an illegal syndicate acts on behalf of the population of bureaucrats and is able to exploit its strategic advantage to increase total corruption proceeds. 
Notice that, given $C^{\prime}(\cdot)>0$ and $\bar{\lambda}^{\prime}(\cdot)<0$

$$
g^{\prime}(\gamma)=1-\alpha C^{\prime}(\bar{\lambda}(\gamma)) \bar{\lambda}^{\prime}(\gamma)>0
$$

and that given $\lambda_{\mathrm{NC}}>\lambda_{\min }, g(0)<0$. Consider the following three cases:

(1) $g(\tilde{\gamma})>0$. Given $\widehat{\gamma} \leqslant \tilde{\gamma}$ the equilibrium condition it is obtained by substituting $\lambda(\gamma)=\bar{\lambda}(\gamma)$ into $(13)$ to obtain

$$
\widehat{\gamma}=\alpha[C(\bar{\lambda}(\widehat{\gamma}))-k] \Longleftrightarrow g(\widehat{\gamma})=0 .
$$

Given $g(0)<0, g(\tilde{\gamma})>0$, and $g^{\prime}(\gamma)>0$, a unique equilibrium exists with $\widehat{\gamma}<\tilde{\gamma}$.

(2) $g(\tilde{\gamma})=0$. Given $\widehat{\gamma} \leqslant \tilde{\gamma}$ the equilibrium condition is obtained by substituting $\lambda(\gamma)=\bar{\lambda}(\gamma)$ into $(13)$ to obtain

$$
g(\widehat{\gamma})=0 .
$$

Given $g(0)<0, g(\tilde{\gamma})=0$, and $g^{\prime}(\gamma)>0$, a unique equilibrium exists with $\widehat{\gamma}=\tilde{\gamma}$.

(3) $g(\tilde{\gamma})<0$. Suppose an equilibrium exists with $\widehat{\gamma}<\tilde{\gamma}$. Then it is obtained by substituting $\lambda(\gamma)=\bar{\lambda}(\gamma)$ into (13) to obtain

$$
g(\widehat{\gamma})=0 .
$$

Given $g(\tilde{\gamma})<0$ and $g^{\prime}(\gamma)>0$ a contradiction arises to the hypothesis that an equilibrium exists with $\widehat{\gamma}<\tilde{\gamma}$. We will now show that a unique equilibrium exists with $\widehat{\gamma}=\tilde{\gamma}$.

Let

$$
\widetilde{p}=\frac{\tilde{\gamma}}{\alpha(C(\bar{\lambda}(\tilde{\gamma}))-k)} \in(0,1)
$$

and consider the following strategy prof le:

(C) The constituency in stage 1 plays $\bar{\lambda}(\tilde{\gamma})$ with probability $\tilde{p}$ and $\lambda_{\min }$ with probability $1-\tilde{p}$.

(B) Bureaucrats with corruption entry costs less than or equal to $\tilde{\gamma}$ pay the corruption entry cost in stage 1 . If the realization of the constituency's strategy in stage 2 is $\lambda_{\min }$ no bureaucrat arranges a corrupt transaction in stage 3 ; If the realization of the constituency's strategy in stage 2 is $\bar{\lambda}(\tilde{\gamma})$ all bureaucrats who had paid the corruption entry cost arrange a corrupt transaction in stage 3 .

Given the expected payoff from paying the corruption entry cost in stage 1 to a risk neutral bureaucrat is $\tilde{\gamma}$, all bureaucrats with corruption cost less than or equal to $\tilde{\gamma}$, a fraction $\tilde{\gamma}$ of the total, will pay the corruption entry cost. Given $\bar{\lambda}(\tilde{\gamma})$ and $\lambda_{\min }$ are the constituency's best responses to $\tilde{\gamma}$, the previous strategies constitute an equilibrium. Observing that the best response of the constituency is $\Sigma\left(\left\{\lambda_{\min }, \bar{\lambda}(\tilde{\gamma})\right\}\right)$ and that $\tilde{p}$ is the only probability of playing $\bar{\lambda}(\tilde{\gamma})$ such that the aggregate best response of the bureaucrats is $\tilde{\gamma}$ shows that no other equilibrium exists. 
(2) Suppose $\lambda_{\mathrm{NC}} \leqslant \lambda_{\min }$. From lemma 1 we get $\widehat{\lambda}=\lambda_{\mathrm{NC}}$. Substituting $\widehat{\lambda}=\lambda_{\mathrm{NC}}$ into (13) we get $\widehat{\gamma}=0$.

Example 1. Suppose $V(\lambda)=\log \lambda, C(\lambda)=\lambda, \lambda_{c}=0.1, \mu=0.5, P^{\mathcal{B}}=P^{\mathcal{P}}=0.05$, and $\alpha=1$. This implies that $k=0.15$ and that $\lambda_{\min }=k=0.15<\lambda_{\mathrm{NC}}=1$. Notice also

$$
\bar{\lambda}(\gamma)=1-\gamma>\lambda_{\min } \Longleftrightarrow \gamma<1-\lambda_{\min }=0.85=\gamma^{\prime}
$$

and that $\tilde{\gamma}=0.53122$. From (11) we have

$$
\gamma(\lambda)= \begin{cases}\lambda-k & \text { if } \lambda \geqslant \lambda_{\min }=0.15 \\ 0 & \text { if } \lambda<\lambda_{\min }=0.15\end{cases}
$$

and from lemma 1 the best response of the constituency is $\bar{\lambda}(\gamma)=1-\gamma$ for $\gamma<$ $\tilde{\gamma}=0.53122$, any probability distribution on $\left\{\lambda_{\min }, 1-\tilde{\gamma}\right\}=\{0.2,0.46878\}$ when $\gamma=\tilde{\gamma}=0.53122$, and $\lambda_{\min }=0.15$ when $\gamma>\tilde{\gamma}=0.53122$. Figure 1 depicts this situation by plotting the best response of the constituency to the aggregate strategy of the bureaucrats, $\lambda(\gamma)$, and the aggregate best response of the bureaucrats to the strategy of the constituency, $\gamma(\lambda)$, respectively. Straightforward computations show that the equilibrium is

$$
\widehat{\lambda}=0.575, \quad \widehat{\gamma}=0.425 \text {. }
$$

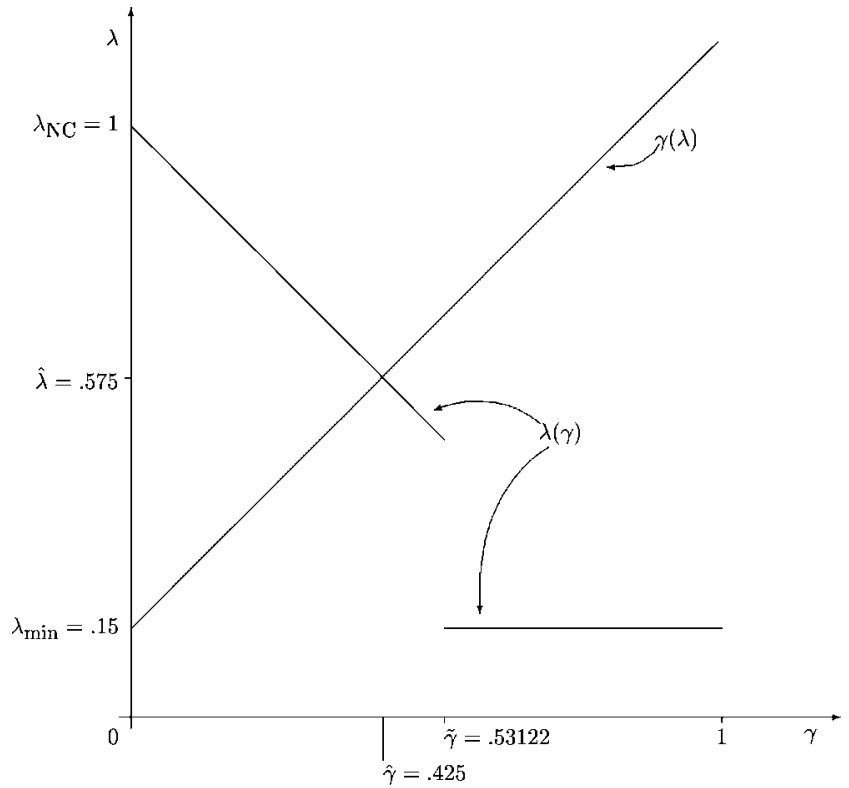

Figure 1. Competitive corruption in example 1. 


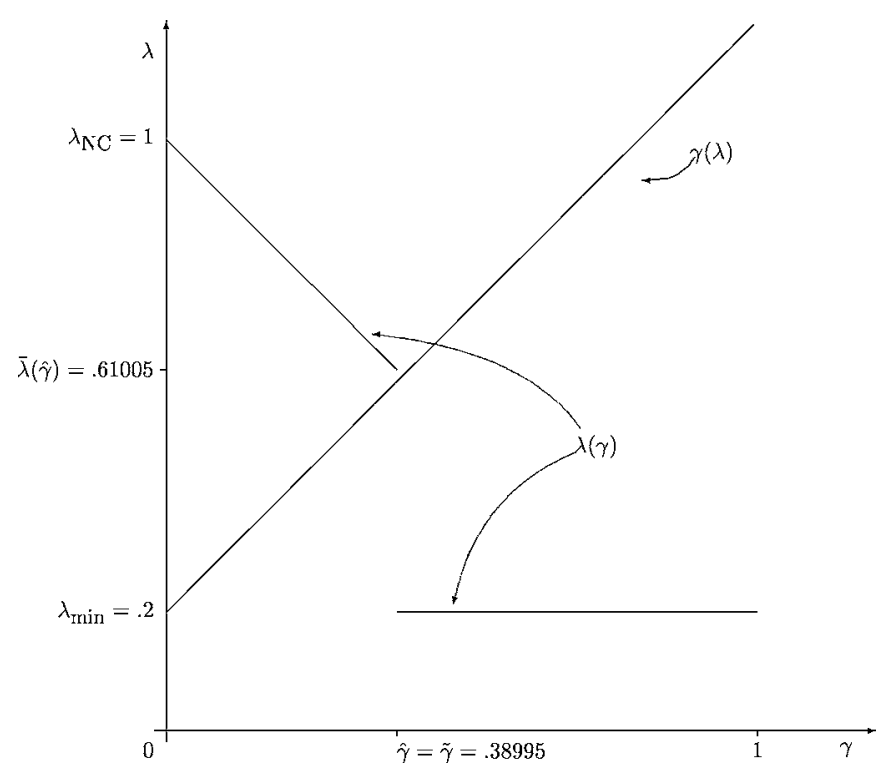

Figure 2. Competitive corruption in example 2.

Example 2. Consider a case in which corruption penalties are higher than in the previous example, $P^{\mathcal{B}}=P^{\mathcal{P}}=0.1$. In this case we would have $k=0.2, \lambda_{\min }=k=0.2<$ $\lambda_{\mathrm{NC}}=1$,

$$
\bar{\lambda}(\gamma)=1-\gamma>\lambda_{\min } \Longleftrightarrow \gamma<1-\lambda_{\text {min }}=0.8=\gamma^{\prime}
$$

and $\tilde{\gamma}=0.38995$. From lemma 1 the best response of the constituency is $\bar{\lambda}(\gamma)=(1-\gamma)$ for $\gamma<\tilde{\gamma}=0.38995$, any probability distribution on $\left\{\lambda_{\min }, 1-\tilde{\gamma}\right\}=\{0.3,0.61005\}$ when $\gamma=\tilde{\gamma}=0.38995$, and $\lambda_{\min }=0.2$ when $\gamma>\tilde{\gamma}=0.38995$. Also notice that $\bar{\lambda}(\tilde{\gamma})=0.61005$ and that $\tilde{p}=0.95098$. Figure 2 depicts this situation. From proposition 2 equilibrium actions are the following: in stage 1 all bureaucrats with corruption entry cost less than or equal to $\widehat{\gamma}=\tilde{\gamma}=0.38995$ (a fraction $\widehat{\gamma}=\tilde{\gamma}=0.38995$ ) pay the corruption entry cost; In stage 2 the constituency plays $\lambda=0.61005$ with probability $\widetilde{p}=0.95098$ and $\lambda=0.2$ with probability $1-\tilde{p}=0.04902$; In stage 3 if the realization of the constituency's strategy in stage 2 was $\lambda=0.61005$, all bureaucrats who paid the corruption entry cost in stage 1 arrange corrupt transactions, whereas if the realization was $\lambda=0.2$ no bureaucrat arranges a corrupt transaction. This implies that the expected value of $\lambda$ is

$$
\widetilde{p} \bar{\lambda}(\widetilde{\gamma})+(1-\widetilde{p}) \lambda_{\min }=0.95098 \times 0.61005+0.04902 \times 0.2=0.58995 .
$$

Example 3. If corruption penalties are substantially higher than in the previous examples, $P^{\mathcal{B}}=P^{\mathcal{P}}=1, \lambda_{\min }=1.1>\lambda_{\mathrm{NC}}=1$ and even the highest $\lambda$ that could possibly be chosen in equilibrium, i.e., the $\lambda$ that would be chosen if no corruption existed, $\lambda_{\mathrm{NC}}$, is 


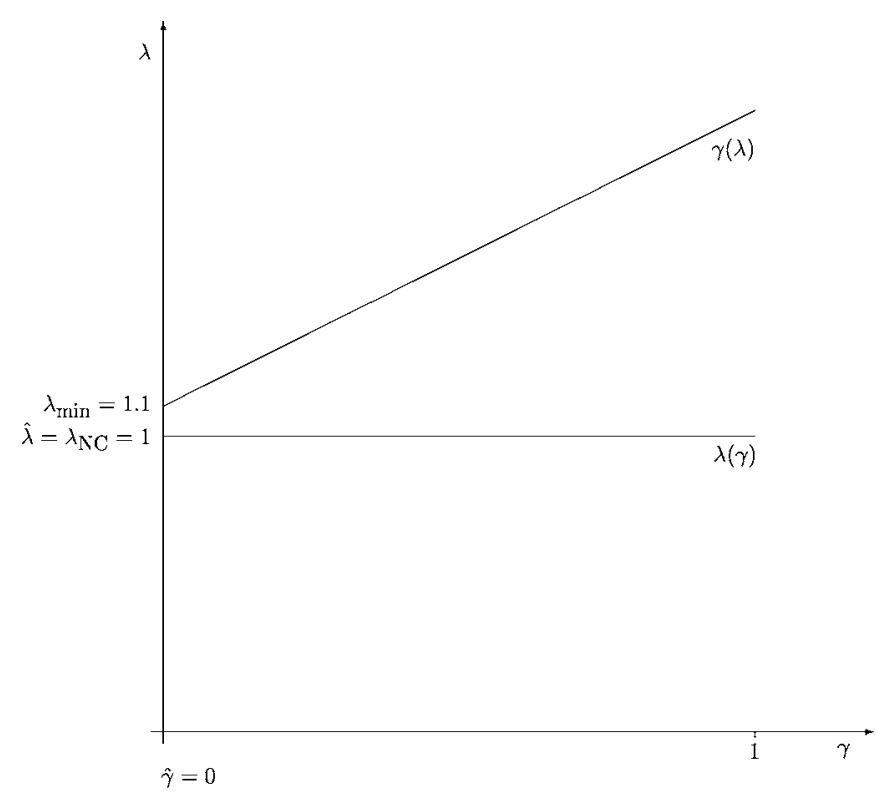

Figure 3. Competitive corruption in example 3.

not suff ciently high for it to be worthwhile for any bureaucrats to be corrupt (as it is strictly smaller than $\lambda_{\min }$ ). This case is depicted in f gure 3 (notice that from lemma 1 when $\lambda_{\min }>\lambda_{\mathrm{NC}}$ the best response of the constituency is $\lambda_{\mathrm{NC}}$ for any value of $\gamma$ ) and in equilibrium $\widehat{\gamma}=0$ and $\widehat{\lambda}=\lambda_{\mathrm{NC}}=1$.

\section{Organized corruption}

In subsection 3.2 we have analyzed the optimal response of the constituency to a given fraction $\gamma$ of bureaucrats having paid the corruption entry cost in stage 1. A higher value of $\gamma$ was then argued to have a negative impact on the marginal value of activity levels and the constituency's response to it was therefore lower activity levels. Given lower activity levels lead to lower returns from corruption, it seems natural to ask if corrupt bureaucrats could improve upon the outcome under competitive corruption and how the equilibrium would differ from the case of competitive corruption. The goal of this section is to turn to these questions.

Many cases have been documented in which corruption has been centrally organized over extended periods of time with examples ranging from Italy, to the Soviet Union, Korea, and Japan. To study the possibility of bureaucrats coordinating their corruption decisions, we assume that an illegal syndicate of the population of bureaucrats centralizes the decision of how many corrupt transactions to arrange with the goal to maximize the total payoffs to the bureaucrats. We refer to this situation as organized 
corruption following Schelling's [14] def nition of organized crime as an organization that has monopoly power over illegal activities.

In this section we will focus on the case in which a syndicate centralizes corruption decisions and sets the fraction of bureaucrats who, by paying the corruption entry cost prior to the constituency setting activity levels, prepare to arrange corrupt transactions is stage $3 .{ }^{9}$ We regard this simple setup as a way to characterize a more realistic situation in which the syndicate's time horizon exceeds the ones of the politically appointed constituency's representatives and in which the syndicate is in a position to establish a reputation for playing the Stackelberg strategy, i.e., for setting an aggregate level of corruption that maximizes total net prof $t$ from corruption given that the constituency plays a best response to it. ${ }^{10}$

Under organized corruption the illegal syndicate acting on behalf of bureaucrats takes into account the constituency's best response. In other words, the syndicate chooses a $\beta^{O}$ such that all (and only) bureaucrats with $\beta<\beta^{O}$ pay the corruption entry cost in stage 1 (and, in equilibrium, will arrange corrupt transactions in stage 3 ) so as to maximize their aggregate payoff ${ }^{11}$

$$
\max _{\beta \in[0,1]} \int_{0}^{\beta}[\alpha(C(\lambda(\beta))-k)-s] \mathrm{d} s .
$$

To guarantee that an equilibrium exists we make the assumption that, whenever indifferent between two values of $\lambda$, the constituency will choose the higher one. The following proposition provides a comparison of equilibrium corruption under organized and competitive corruption.

Proposition 3. Equilibrium corruption is lower and equilibrium bribe is higher under organized than under competitive corruption.

Proof. We will analyze three different cases:

(1) $\lambda_{\mathrm{NC}}>\lambda_{\min }$ and $\widehat{\gamma}<\tilde{\gamma}$;

(2) $\lambda_{\mathrm{NC}}>\lambda_{\min }$ and $\widehat{\gamma}=\tilde{\gamma}$;

(3) $\lambda_{\mathrm{NC}} \leqslant \lambda_{\min }$.

${ }^{9}$ It is essential to assume that the fraction of bureaucrats who pay the corruption entry cost chosen by the syndicate is observed by the constituency before it decides on required activity levels. If the constituency did not observe this, the equilibrium would be identical to the case of competitive corruption. With respect to this assumption, see footnote 10 .

${ }^{10}$ If the syndicate's horizon is longer than the constituency's, the simple sequential model we propose provides predictions in line with the results of the reputation literature initiated by Fudenberg and Levine [7]. In particular notice that even if the constituency observed a noisy signal on the number of corrupt transactions arranged in stage 3 of every period (and not directly the fraction of bureacrats who pay the corruption cost in stage 1 , which is admittedly more diff cult to observe) a suff ciently patient syndicate would be able to achieve a payoff almost as high as the Stackelberg payoff.

${ }^{11}$ In the paper we assume that the syndicate acts on behalf of all bureacrats but the same results hold if the syndicate only centralizes the decisions of a fraction of bureaucrats. 
(1) In this case the equilibrium indifferent type of bureaucrat under competitive corruption is def ned as follows

$$
\alpha[C(\bar{\lambda}(\widehat{\beta}))-k]-\widehat{\beta}=0 .
$$

Assume that the solution to the syndicate's maximization problem is $\beta^{O}<\tilde{\gamma}$. This implies that the syndicate maximizes

$$
F(\beta)=\int_{0}^{\beta}[\alpha(C(\bar{\lambda}(\beta))-k)-s] \mathrm{d} s .
$$

Notice that $F(0)=0$, and, given $\bar{\lambda}(1)=0, F(1)<0$.

When $\lambda_{\mathrm{NC}}>\lambda_{\min }$,

$$
\alpha[C(\bar{\lambda}(0))-k]>0 \quad \text { and } \quad F^{\prime}(0)=\alpha[C(\bar{\lambda}(0))-k]>0 .
$$

Given $F(0)=0$ and $F(1)<0$ this implies that the solution to (14) has to be interior and therefore has to be characterized by the following frst order condition:

$$
\alpha C\left(\bar{\lambda}\left(\beta^{O}\right)\right)-\alpha k-\beta^{O}+\alpha C^{\prime}\left(\bar{\lambda}\left(\beta^{O}\right)\right) \bar{\lambda}^{\prime}\left(\beta^{O}\right) \beta^{O}=0 .
$$

In the following we will show that any value of $\beta^{O}$ satisfying (16) is strictly lower than the indifferent type of bureaucrat under competitive corruption. ${ }^{12}$ Subtracting (15) from (16) we obtain:

$$
\alpha\left[C\left(\bar{\lambda}\left(\beta^{O}\right)\right)-k\right]-\beta^{O}-\alpha[C(\bar{\lambda}(\widehat{\beta}))-k]+\widehat{\beta}=-\alpha\left[C^{\prime}\left(\bar{\lambda}\left(\beta^{O}\right)\right) \bar{\lambda}^{\prime}\left(\beta^{O}\right) \beta^{O}\right] .
$$

Given $C^{\prime}\left(\bar{\lambda}\left(\beta^{O}\right)\right)>0$ and $\bar{\lambda}^{\prime}\left(\beta^{O}\right)<0, C^{\prime}\left(\bar{\lambda}\left(\beta^{O}\right)\right) \bar{\lambda}^{\prime}\left(\beta^{O}\right) \beta^{O}<0$. Then

$$
\alpha\left[C\left(\bar{\lambda}\left(\beta^{O}\right)\right)-k\right]-\beta^{O}>\alpha[C(\bar{\lambda}(\widehat{\beta}))-k]-\widehat{\beta}
$$

and given $\alpha[C(\bar{\lambda}(\beta))-k]-\beta$ is decreasing in $\beta$, (17) implies $\beta^{O}<\widehat{\beta}$ and $\gamma^{O}<\widehat{\gamma}$. The previous inequality also implies that $\lambda^{O}=\lambda\left(\gamma^{O}\right)>\lambda(\widehat{\gamma})=\widehat{\lambda}$. Because by (2) the equilibrium bribe is increasing in $\lambda$, the equilibrium bribe will be higher under organized than under competitive corruption. Given $\beta^{O}<\widehat{\beta}$ and we are dealing with the case in which $\widehat{\gamma}=\widehat{\beta}<\tilde{\gamma}$, the assumption that $\beta^{O}<\tilde{\gamma}$ is satisf ed.

(2) Given $\widehat{\gamma}=\tilde{\gamma}$, to prove the claim it suff ces to show that $\beta^{O}>\tilde{\gamma}$ is impossible. Suppose $\beta^{O}=\bar{\gamma}>\widetilde{\gamma}$. From lemma 1 we have that $\lambda\left(\beta^{O}\right)=\lambda_{\min }$ which in turn implies that no bureaucrat that paid the corruption entry cost in stage 1 will engage in corrupt transactions in stage 3 and therefore the payoff to the syndicate is

$$
-\int_{0}^{\bar{\gamma}} s \mathrm{~d} s=-\frac{\bar{\gamma}^{2}}{2}
$$

12 This argument makes it unnecessary to study the second order condition. Given the solution has to be a stationary point of $F(\beta)$, showing that all stationary points are lower than the equilibrium indifferent type under competitive corruption is suff cient to prove the claim of the proposition. 
Setting $\beta^{O}=\tilde{\gamma}$, on the other hand implies that $\lambda\left(\beta^{O}\right)=\bar{\lambda}(\widetilde{\gamma})>\lambda_{\min }$. This in turn implies that all bureaucrats who paid the corruption entry cost in stage 1 will be corrupt in stage 3 and the net payoff to the syndicate will be

$$
\int_{0}^{\tilde{\gamma}}[\alpha(C(\bar{\lambda}(\widetilde{\gamma}))-k)-s] \mathrm{d} s=\int_{0}^{\tilde{\gamma}} \alpha(C(\bar{\lambda}(\widetilde{\gamma}))-k) \mathrm{d} s-\frac{\tilde{\gamma}^{2}}{2} .
$$

Given $\int_{0}^{\tilde{\gamma}} \alpha(C(\bar{\lambda}(\widetilde{\gamma}))-k) \mathrm{d} s>0$ and $\tilde{\gamma}<\bar{\gamma}$, setting $\beta^{O}=\tilde{\gamma}$ is a prof table deviation from $\beta^{O}=\bar{\gamma}>\tilde{\gamma}$ and a contradiction is obtained.

(3) When $\lambda_{\mathrm{NC}}>\lambda_{\min }$, from lemma $1 \bar{\lambda}(\gamma)=\lambda_{\mathrm{NC}}$ for $\gamma \in[0,1]$ and implies that $F^{\prime}(\gamma)=\alpha[C(\bar{\lambda}(\gamma))-k]=\alpha\left[C\left(\lambda_{\mathrm{NC}}\right)-k\right]<0$ for $\gamma \in[0,1]$. Given this the solution to (14) is $\beta^{O}=0$ and $\gamma^{O}=\widehat{\gamma}=0$ and $\lambda^{O}=\widehat{\lambda}=\lambda_{\mathrm{NC}}$.

Example 4. Consider the same case as in example 1 and recall that when, $P^{\mathcal{B}}=$ $P^{\mathcal{P}}=0.05, \widehat{\gamma}=0.425<\tilde{\gamma}=0.53122$. From proposition 3 we then know that in the equilibrium of the game with organized corruption all agents with $\beta<\beta^{O}$ will pay the corruption entry cost in stage 1 with $\beta^{O}$ satisfying (16), i.e.,

$$
1-\beta^{O}-0.15-\beta^{O}-\beta^{O}=0 \quad \Longleftrightarrow \quad \beta^{O}=\gamma^{O}=0.28333<\widehat{\gamma}=0.425
$$

and this implies that $\lambda^{O}=0.71667>\widehat{\lambda}=0.575$.

Example 5. Consider the case as of example 2 in which $\widehat{\gamma}=\tilde{\gamma}=0.38995$. With organized corruption from (16) we get

$$
1-\beta^{O}-0.2-\beta^{O}-\beta^{O}=0 \quad \Longleftrightarrow \quad \beta^{O}=0.26667 \text {. }
$$

Given $\beta^{O}=0.26667<\tilde{\gamma}=0.38995, \beta^{O}$ is a solution to the syndicate's maximization problem and in the equilibrium with organized corruption a lower fraction of bureaucrats pay the corruption entry cost than in the equilibrium with competitive corruption

$$
\gamma^{O}=0.26667<\widehat{\gamma}=0.38995
$$

and this implies that $\lambda^{O}=0.7333$ which is higher than the expected value of $\lambda$ in the competitive corruption equilibrium, 0.58995 .

Example 6. In the case of example 3, in the equilibrium with organized corruption we get $\gamma^{O}=\widehat{\gamma}=0$ and $\lambda^{O}=\widehat{\lambda}=\lambda_{\mathrm{NC}}=1$.

As the proof of proposition 3 and the previous examples should have clarif ed, when $\widehat{\gamma}<\widetilde{\gamma}$, equilibrium under organized corruption is always strictly lower than equilibrium with competitive corruption; but when $\widehat{\gamma}=\tilde{\gamma}$, the equilibrium value of $\gamma$ with organized corruption may be strictly lower than $\widehat{\gamma}$ (as in the example with $P^{\mathcal{B}}=P^{\mathcal{P}}=0.1$ ) but it can also be identical to it, as one can readily verify for the case in which $P^{\mathcal{B}}=P^{\mathcal{P}}=0.2$.

Proposition 3 rests on the following argument. Increasing the number of corrupt bureaucrats has an obvious direct effect that increases total corruption revenue. Because 


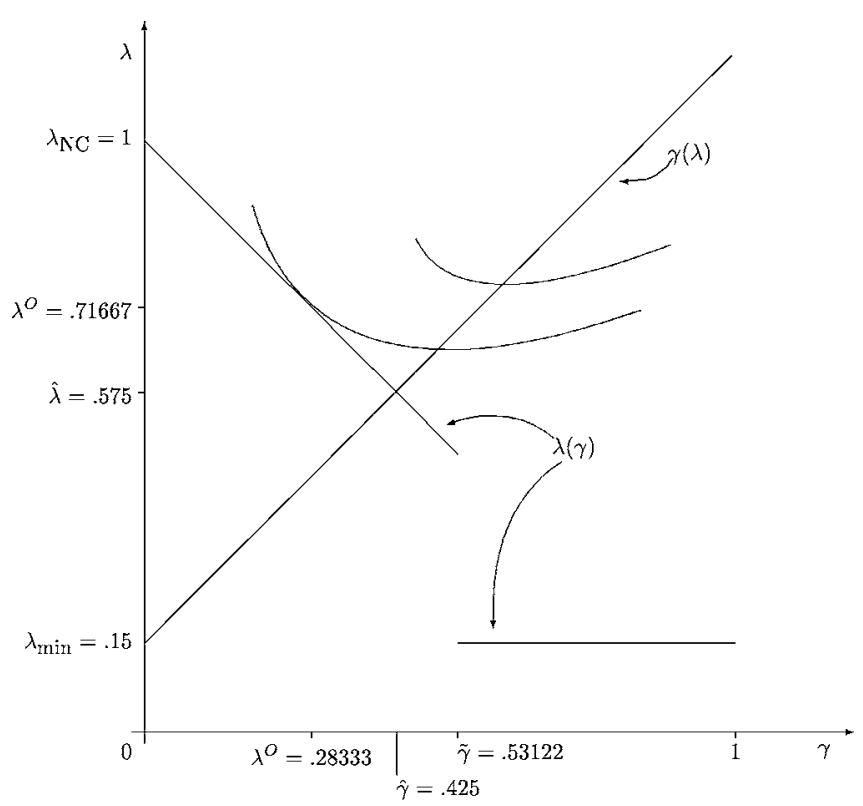

Figure 4. Organized corruption in example 4.

higher corruption also implies a response on the part of the constituency that decreases the activity levels, and, therefore, the prof $t$ per corrupt transaction, increasing corruption also has an indirect effect that decreases total corruption revenue. Proposition 3 shows that when bureaucrats centralize the corruption decision, they internalize the indirect effect of corruption on total corruption revenue (much like a monopolist internalizes the pecuniary externalities of supply decisions) and restrict the number of corrupt transactions as compared to the case with competitive corruption.

The difference between organized and competitive corruption is shown in f gure 4 representing the case in which $P^{\mathcal{B}}=P^{\mathcal{P}}=0.05$ in examples 2 and 4 . Figure 4 reproduces f gure 1 but it adds a map of isoprof $t$ curves for the bureaucrats. Consider a point on the bureaucrats' aggregate best response, $(\lambda, \gamma(\lambda))$. Since $\gamma(\lambda)$ is the aggregate best response to $\lambda,{ }^{13}$ it is straightforward to recognize that for the bureaucrats to have the same aggregate prof ts with corruption levels different from $\gamma(\lambda)$ (that is to say for levels of corruption that are either strictly larger or strictly smaller than $\gamma(\lambda)$ and, therefore, suboptimal given $\lambda$ ), it is necessary that $\lambda^{\prime}>\lambda$, so that the isoprof t curves have a minimum at their intersection with $\gamma(\lambda)$. Given higher isoprof $t$ curves are associated to higher aggregate prof ts for the bureaucrats, it is easy to recognize that, under organized corruption, the bureaucrats will choose the point on the constituency's best response function $\lambda(\gamma)$ that lies on the highest isoproft curve, e.g., point $\gamma^{O}<\widehat{\gamma}$ in

${ }^{13}$ Notice that $\gamma(\lambda)$ not only describes the response of individual agents to a given $\lambda$, but is also the level of corruption that maximizes total prof ts from corruption for a given $\lambda$, i.e., it is the syndicate's best response to a given $\lambda$. 


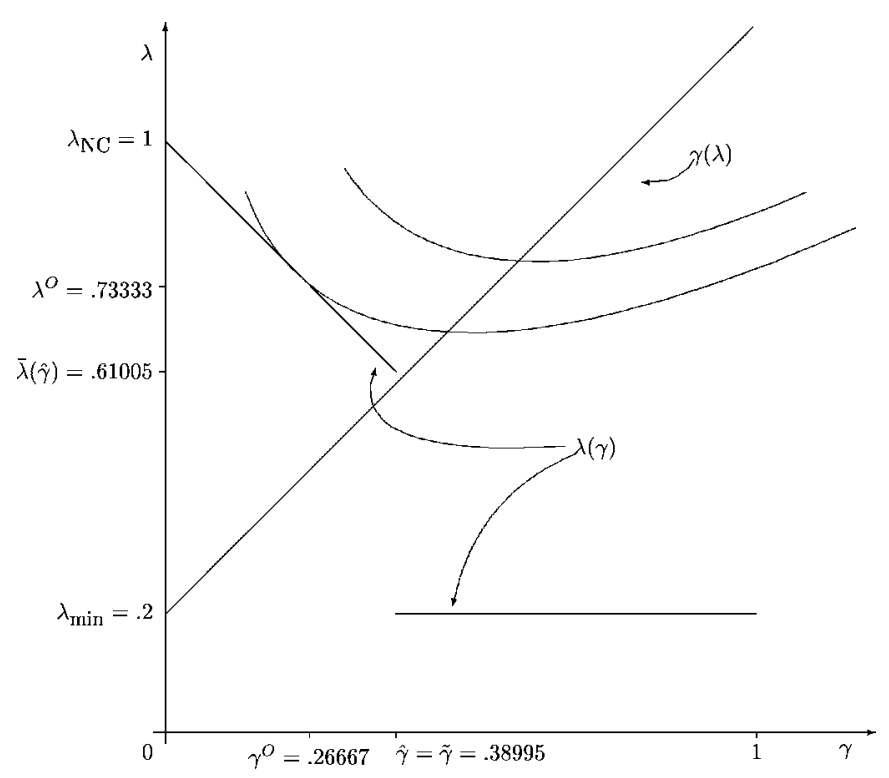

Figure 5. Organized corruption in example 5.

f gure 4. Figure 5 depicts the comparison between organized and competitive corruption of example 5.

Proposition 3 has important implications for the way in which the constituency's optimal behavior depends on the degree of centralization of corruption. Proposition 3 shows that, if the bureaucrats anticipate that the constituency behaves optimally, the optimal activity level is higher when corruption is organized than when it is competitive (see examples 4 and 5).

Finally, notice that from (2) the equilibrium bribe is increasing in the equilibrium activity level $\lambda$, and $\lambda^{O} \geqslant \hat{\lambda}$ implies that equilibrium bribes under organized will be no lower than under competitive corruption.

The result of proposition 3 is similar to Shleifer and Vishny's [15]. In their model a sequence of independent monopolistic corrupt bureaucrats face a market that demands their services and organized corruption serves to solve the problem of multiple marginalization. In our model, the optimal regulatory response of the constituency implies that the unit value of corrupt transaction is higher when total corruption is lower and organized corruption serves to optimally trade off corruption revenue base against corrupt transaction unit prof $\mathrm{t}$, so that corruption is lower than under competitive corruption.

Apart from the previous similarity, as was mentioned in the introduction, the comparisons in proposition 3 of equilibrium corruption under organized and competitive corruption are opposite to the ones proposed by Shleifer and Vishny [15]. According to Shleifer and Vishny [15] under competitive corruption the excessive demands of individual bureaucrats price many potential bribers out of the market so that bribes are higher and total corruption is lower. To assess the differences between the predictions of the two models, it is important to highlight the fact that Shleifer and Vishny [15] do 
not allow for bureaucrat heterogeneity and assume that $100 \%$ of transactions are corrupt regardless of whether bureaucrats collude or not. Given these assumptions, the total number of corrupt transactions turns out to be higher when bureaucrats collude (as in this case they charge a lower bribe). By contrast, we introduce bureaucrat heterogeneity, and explicitly focus on the fraction of transactions which turn out to be corrupt in equilibrium, and, while we endogenize the activity levels, we assume that total number of transactions (corrupt or not) is f xed.

Because the two models focus their attention on different issues, we regard them as providing complementary rather than conf icting explanations. But the different testable implications of the comparisons between organized and competitive corruption of the two models allow empirical studies to assess their relative merits. While we are not aware of systematic data on the degree of centralization of corruption, tentative evidence is provided by anecdotal testimony on the collapse of the Soviet Union. Several authors have argued that the Communist Party of the Soviet Union as well as the KGB provided a central structure for corrupt exchanges, and that the end of the Soviet Union has given way to a much less centralized organization of corruption in Russia. ${ }^{14}$ According to survey data by the International Country Risk Guide, this transition has witnessed a signif cant surge in corruption, ${ }^{15}$ a f nding in line with the predictions of this paper.

Before concluding we want to mention that a straightforward implication of proposition 3 is that the equilibrium under organized corruption Pareto dominates the equilibrium with competitive corruption as corruption is lower and corruption prof $t$ is higher. ${ }^{16}$ We believe that such a welfare comparison should be discounted and should not be taken as grounds for sensible policy implication as the model we consider does not keep into account other features of organized corruption that may make it less appealing from the point of view of society, as, for instance, a better technology to decrease the effectiveness of deterrence policies.

\section{Conclusions}

This paper proposes a simple model of bureaucratic corruption and studies the possibility of bureaucrats centralizing corruption decisions. The main goal of the paper is to analyze if an illegal syndicate acting on behalf of the population of bureaucrats is able to improve upon the outcome that obtains when bureaucrats make the decision to be corrupt

${ }^{14}$ See, for example, Shleifer and Vishny [15], Bardhan [2], and Leitzel [11]. In referring to Russian Maf a, Judge Giovanni Falcone claimed that it lacked the unitary structure of sicilian Maf a: "There is no sense in calling something a Maf a when it is not" [6, p. 102].

15 The International Country Risk Guide is a monthly publication of the PRS Group and it includes a rating of corruption risk from 0 (highest risk of corruption) to 6 (lowest risk of corruption). According to data published by the International Country Risk Guide and assembled by the IRIS Center (University of Maryland), Russia's corruption rating in 1985-1991 was 4, went down to 3.5 in 1992, and to 3 in 1993-1995. Leitzel [11] shares the view the corruption in Russia increased as a consequence of the reforms that followed the failed putsch of August 1991.

16 The same result is obtained by Shleifer and Vishny [15]. 
individually and what implications this may have on the equilibrium level of corruption. Our main $\mathrm{f}$ ndings can be summarized as follows:

1. When corruption decisions are centralized (organized corruption) corruption is lower and bribes are higher than when corruption decisions are decentralized (competitive corruption)

2. When corruption decisions are centralized, the constituency's activity levels are higher than when corruption decisions are decentralized.

The results rely on the assumption that the constituency affected by corruption responds optimally to it in a way that reduces the revenue per corrupt transaction unit. When this element is taken into account, i.e., when it is clarif ed that corruption may extinguish its culture medium, it is easy to recognize that corruption revenue is not monotonically increasing in corruption level. When bureaucrats individually decide whether to be corrupt or not, they disregard the indirect effect that their decision to be corrupt has on other bureaucrats' corruption revenue. Under organized corruption, on the other hand, the effect that increasing corruption has on total corruption revenue through the constituency's response is internalized and the illegal syndicate acts in such a way as to restrain the total number of corrupt transactions as compared to the case of competitive corruption.

In an attempt to focus on the difference between competitive and organized corruption, this paper made use of a simple one-period model. The results we propose can be generalized to more realistic intertemporal settings but an interesting issue is the additional implications the results of this paper can have on the dynamics of corruption. Making use of the results of this paper and of a related paper, Celentani and Ganuza [4], that studies explicitly the dynamics of corruption with overlapping generations of corruptible agents, it is possible to make the following testable predictions on the transition from organized to competitive corruption or vice versa.

(i) If a competitive setting prevails when corruption is in the organized corruption steady state, corruption will follow a nonmonotonic path: its level will $\mathrm{frst}$ jump up and will then converge down to the new higher steady state;

(ii) If organized corruption suddenly prevails at the competitive corruption steady state, corruption will monotonically decrease down to the new lower steady state.

The lack of reliable data on the degree of centralization of corruption decisions makes the task of empirically testing the predictions of our results a signif cant challenge. But available evidence on post communist Russia provides encouraging if tentative support to our predictions.

\section{Acknowledgments}

Marco Celentani acknowledges the f nancial support of DGES of MEC (Spain) under project PB98-00024 and of MURST (Italy) under project \# 9813282901_004. Juan-José 
Ganuza acknowledges the $\mathrm{f}$ nancial support of the Spanish Ministry of Science and Technology under project BEC2000-1026. We are grateful to the referees for many helpful comments.

\section{References}

[1] A. Ades and R. Di Tella, Rents, competition, and corruption, American Economic Review 89(4) (1999) 982-993.

[2] P. Bardhan, Corruption and development: A review of issues, Journal of Economic Literature 35 (1997) 1320-1346.

[3] J. Cartier-Bresson, Corruption networks, transaction security and illegal social exchange, Political Studies 45 (1997) 463-476.

[4] M. Celentani and J.-J. Ganuza, Corruption and the Hadleyburg effect, Universitat Pompeu Fabra, Department of Economics, Working Paper No. 382 (1999).

[5] M. Celentani and J.-J. Ganuza, Corruption and competition in procurement, European Economic Review (2002) 1273-1303.

[6] G. Falcone and M. Padovani, Men of Honour: The Truth about the Mafi (Warner Books, London, 1993).

[7] D. Fudenberg and D.K. Levine, Reputation and equilibrium selection in games with a single patient player, Econometrica 57 (1989) 759-778.

[8] A. Goudie and D. Stasavage, Corruption: the issues, OECD Technical Papers, No. 122 (1997).

[9] A. Krueger, The political economy of the rent-seeking society, American Economic Review 64 (1974) 291-303.

[10] J.-J. Laffont and N'Guessan, Competition and corruption in an agency relationship, Journal of Development Economics 60 (1999) 271-295.

[11] J. Leitzel, Corruption and organized crime in the Russian transition, Mimeo, University of Chicago (1998).

[12] P. Mauro, Corruption and growth, Quarterly Journal of Economics 110(3) (1995) 681-712.

[13] P. Mauro, Corruption and the composition of government expenditure, Journal of Public Economics 69(2) (1998) 263-279.

[14] T. Schelling, What is the business of organized crime?, Journal of Public Law 20 (1971). Reprinted in: T. Schelling, Choice and Consequence (Harvard University Press, Cambridge, MA, 1984) pp. 179-194.

[15] A. Shleifer and R.W. Vishny, Corruption, Quarterly Journal of Economics 108 (1993) 599-617.

[16] G. Tullock, The welfare costs of tariffs, monopoly, and theft, Western Economic Journal 5 (1967) 224-232. 